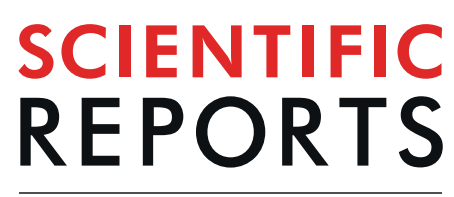

natureresearch

\title{
OPEN Transcriptome analysis reveals the protection mechanism of proanthocyanidins for Saccharomyces cerevisiae during wine fermentation
}

\author{
Jingyuan $\mathrm{Li}^{1}$, Kaili Zhu ${ }^{1}$ \& Hongwei Zhao ${ }^{2,3 *}$ \\ Grape-derived proanthocyanidins could act as a protector against various environmental stresses \\ for Saccharomyces cerevisiae during wine fermentation, resulting in the increased physiological \\ activity, fermentation efficiency and improved wine quality. In order to explore the possible protection \\ mechanism of proanthocyanidins globally, RNA-seq analysis for wine yeast AWRI R2 cultivated \\ with $0 \mathrm{~g} / \mathrm{L}$ (group A), $0.1 \mathrm{~g} / \mathrm{L}$ (group B), $1.0 \mathrm{~g} / \mathrm{L}$ (group C) proanthocyanidins were applied in this \\ study. Differentially expressed genes were enriched into six metabolic pathways including vitamin \\ $B_{6}$, thiamine, amino acids, aminoacyl-tRNA, carbohydrate and steroid based on KEGG enrichment \\ analysis. Four key genes (SNZ2, THI6, THI21 and THI80), participated in the biosynthesis of vitamin \\ $B_{6}$ and thiamine, were up-regulated significantly in proanthocyanidins treated yeast cells and the \\ gene expression levels were verified by RT-qPCR. Yeast cells supplemented with proanthocyanidins \\ performed increased intracellular levels of vitamin $B_{6}$ and thiamine and higher cell viability compared \\ to the control group. In addition, the composition of intracellular fatty acids showed an obvious \\ alternation in proanthocyanidins-treated yeast cells, in which the UFAs content increased whereas the \\ SFA content decreased. In general, we provided an indirect protection effect of proanthocyanidins on \\ the yeast cells to alleviate environmental stresses during wine fermentation.
}

The widely distributed phenolic substances in wine refer to a large group of chemical compounds that impact the sensory quality of red wine, such as color, flavor and astringency ${ }^{1}$. A large number of studies have shown that these phenolic substances have also biological activities of interest to the consumers, such as antioxidant properties, vascular protection and immune-modulatory effects ${ }^{2-5}$. For winemaking, these phenolic compounds are mainly extracted from grape skin, seed, and stem during the maceration and fermentation process ${ }^{6}$. Oenological practices, such as fermentation temperature, maceration time, storage period and yeast strains, are believed to impact the concentration and structure of these chemical compounds $\mathrm{s}^{7-9}$.

As the predominant fraction, proanthocyanidins (PAs) account for more than $50 \%$ of the total wine polyphenols, and have several important roles in the quality of red wine, such as the perception of astringency and taste, aroma release, and color stability, throughout the entire period of fermentation and storage of wine ${ }^{10,11}$.

In addition, PAs could interact with other extracted phenolic compounds and even the fermentation strains, which have been proved to be crucial for the generation of new pigments, color and astringency modifications ${ }^{12,13}$. It has been reported that the interaction of the polysaccharides released by yeast lees with wine polyphenols during aging were crucial for sensory qualities of wines ${ }^{11}$. The extracted polyphenols could be adsorbed by yeast cells by forming the Van der Waals bonds and $\mathrm{H}$-bonds during alcoholic fermentations, which might be a reasonable explanation that the yeasts cells could contribute to the modification of the color of the wine ${ }^{13}$.

\footnotetext{
${ }^{1}$ College of Food Science and Engineering, Qingdao Agricultural University, Qingdao, Shandong Province, 266109, China. ${ }^{2}$ College of Marine Science and Bioengineering, Qingdao University of Science and Technology, Qingdao, Shandong Province, 266042, China. ${ }^{3}$ Shandong Provincial Key Laboratory of Biochemical Engineering, Qingdao, Shandong Province, 266042, China. *email: zhaohw@qust.edu.cn
} 


\begin{tabular}{|l|l|l|l|l|l|}
\hline Sample & length & Reads & Bases & $\begin{array}{l}\text { Q20 } \\
(\%)\end{array}$ & $\begin{array}{l}\text { Q30 } \\
(\%)\end{array}$ \\
\hline A1 & 147.32 & 40811558 & 6012389770 & 95.73 & 88.78 \\
\hline A2 & 147.38 & 62030546 & 9141997823 & 95.75 & 89.47 \\
\hline A3 & 147.53 & 60816138 & 8972430801 & 95.93 & 89.89 \\
\hline B1 & 147.45 & 67018594 & 9882123005 & 95.88 & 89.72 \\
\hline B2 & 147.49 & 55217110 & 8143748191 & 96.01 & 90.04 \\
\hline B3 & 147.41 & 66504646 & 9803127605 & 95.89 & 89.78 \\
\hline C1 & 147.18 & 52310564 & 7699225784 & 95.85 & 89.70 \\
\hline C2 & 147.28 & 55806604 & 8219088002 & 95.85 & 89.69 \\
\hline C3 & 147.21 & 63509302 & 9349372301 & 96.02 & 90.10 \\
\hline
\end{tabular}

Table 1. Quality statistics of clean sequencing data. Q20, Q30 refer to the proportion of base calls with Phred scores $>20$ or 30 in the total bases. Higher scores ensured the base calling accuracy and data quality. Each group has three biological replicates.

Saccharomyces cerevisiae, the preferred microorganism for wine making and bioethanol production, has been exposed simultaneously and sequentially to a variety of environmental stresses during the fermentation process, including high concentration of ethanol, hyperosmotic stress, heat shock, low $\mathrm{pH}$ and nutrients deficiency ${ }^{14-16}$. Cells viability and fermentative behavior will be negatively affected by the stresses that in turn limit the wine production and quality ${ }^{17}$. In order to carry out wine fermentation properly, yeast cells should possess the capacity of rapid stress perceptions and responses to those stress conditions without substantial viability loss ${ }^{14}$. Therefore, to solve these problems, a number of previous studies have been performed in the aspects of robust strain isolation, genetic modification and fermentation process control ${ }^{18-21}$.

It has been reported that phenolic compounds in wine act as a structure-dependent and dose-dependent manner to affect the physiological activities and fermentation performance of yeast and bacterial cells ${ }^{22-24}$. Our previous work showed that PAs could pose obvious enhancement on the metabolism and fermentation efficiency, which has been elucidated from the metabolic level such as the change of glucose transport, the energy and redox homeostasis as well as the activities of rate-limiting enzymes in glycolysis ${ }^{24,25}$. However, the possible molecular mechanisms of the effects of PAs on the yeast fermentation performance have not been fully studied. Next-generation sequencing technologies enables the generation of genomic resources more efficient and cost-effective ${ }^{26}$. Therefore, in this study, we employed RNA-seq as the method to find the differentially expressed key genes and then performed comprehensive and comparative bioinformatics analysis to globally explore the possible protective effects of PAs on the yeast cells during wine fermentation.

\section{Results}

Quality evaluation of sequencing reads. Quality evaluation of sequencing reads needed to be evaluated before the following bioinformatics analysis. As shown in Table 1, a total of 524025062 clean data were generated with an average length of $147 \mathrm{bp}$. The total raw data was deposited in NCBI Gene Expression Omnibus (GEO) public archive database and the accession number is GSE141069. Phred quality score (Q score) was used as an index for the base calling accuracy and calculated by using the FastQC software (v0.10.1) based on Eq. (1):

$$
\mathrm{Q}_{\text {phred }}=-10 \log _{10}(\mathrm{e})
$$

About $90 \%$ of the clean reads in each sample has Phred quality score $>30$, indicating that the base calling accuracy for each group was $99.9 \%$ and met the requirement for the next analysis (Table 1 ).

The Pearson correlation coefficients $\mathrm{R}^{2}$ were calculated to assess the reproducibility of gene expression values across biological replicates, as shown in Supplementary Fig. S1 to Supplementary Fig. S3, the average correlation $\mathrm{R}^{2}$ across the replicates for each group (A, B, C) were $0.969,0.967$ and 0.931 , respectively, indicating that there were no considerable differences between the biological replicates and the data could be used in following analysis.

Differential gene expression analysis. To identify differentially expressed genes, transcriptome analysis was carried out for the yeast cells supplemented with $0 \mathrm{~g} / \mathrm{L}$ (group A), 0.1 g/L (group B) and $1.0 \mathrm{~g} / \mathrm{L}$ (group C) PAs, respectively. As shown in Fig. 1, 389, 1737 and 1566 DEGs were identified by comparative analysis of transcriptome data of group A versus group B, group A versus group C and group B versus group C, respectively, indicating that PAs pose considerable effects on the gene expression level in the yeast cells. Moreover, a dose-dependent increase of DEGs was found in the data comparison (Fig. 2). With the increase of the concentration of PAs from $0.1 \mathrm{~g} / \mathrm{L}$ to $1.0 \mathrm{~g} / \mathrm{L}$ in the fermentation broth, more than 4 times of DEGs were identified in the yeast cells. A total of 48 DEGs were found in all the three comparative groups, which might be considered as the key genes related to the PAs protection mechanism.

GO enrichment analysis of the DEGs. To better understand the functions of the DEGs, gene annotation including homologous protein annotation was performed by using the gene ontology (GO) database. As shown in Fig. 3, the identified DEGs could be classified into three categories including biological process, cellular component and molecular function. 


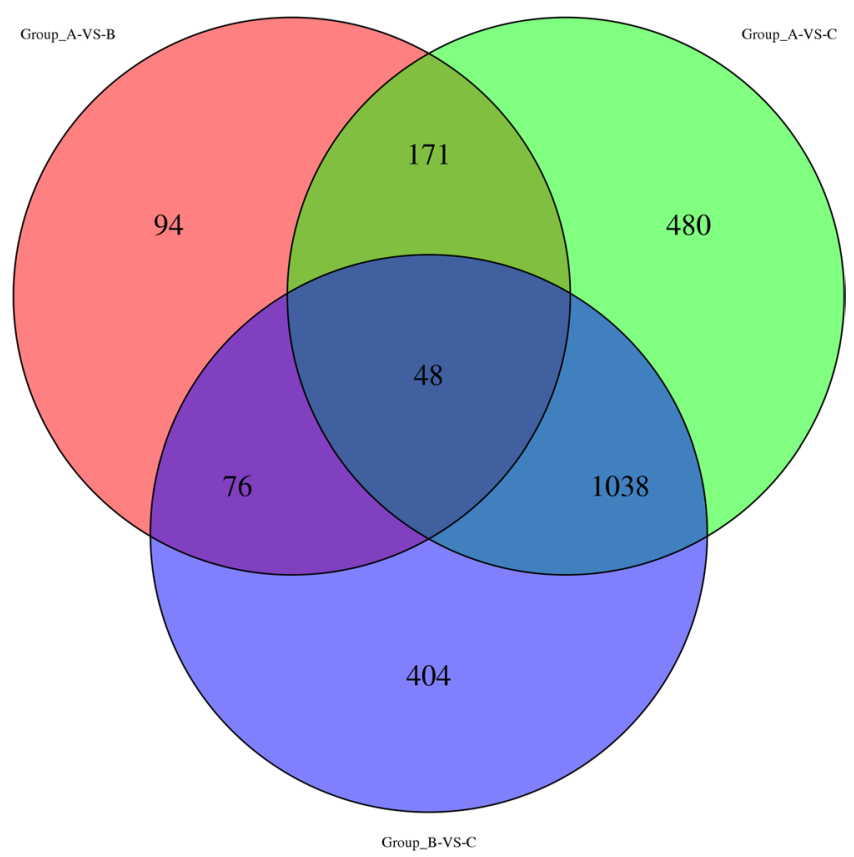

Figure 1. Venn diagrams of transcripts in the three comparative groups marked as group A versus group B, group A versus group C and group B versus group C, respectively.

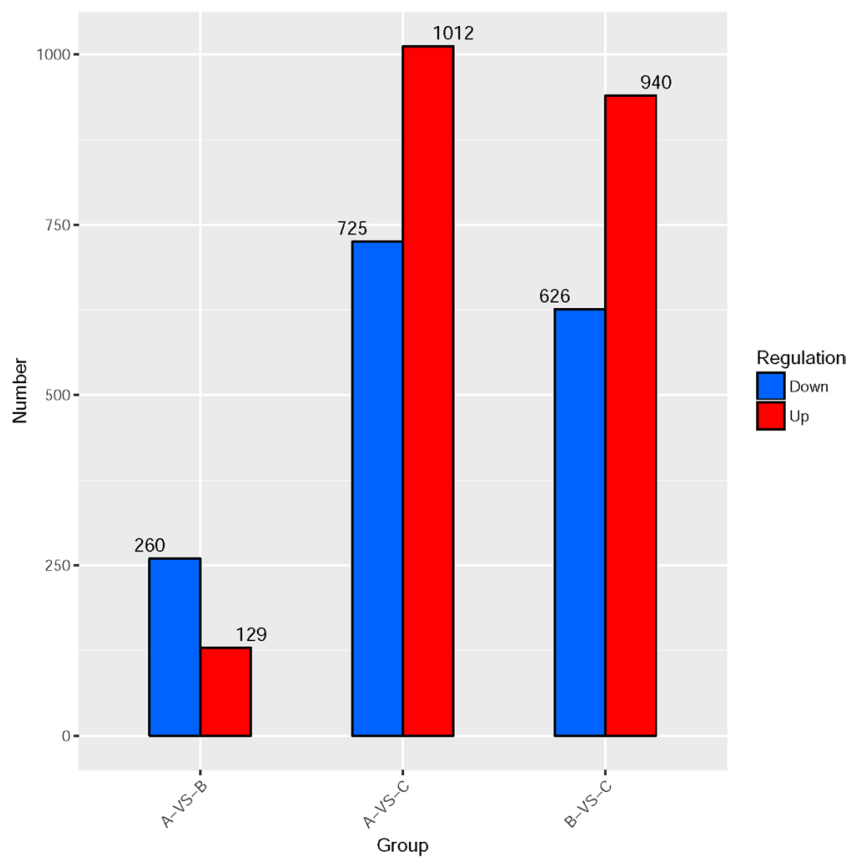

Figure 2. Up or down regulated DEGs in pairwise comparisons marked as group A versus group B, group A versus group $\mathrm{C}$ and group $\mathrm{B}$ versus group $\mathrm{C}$, respectively.

In the cellular component category, DEGs were mainly enriched in the terms related with the structure of cell membrane and organelle; In the biological process group, metabolic process and cellular process were the important functions, especially the thiamine and cellular amino acid metabolic pathways and tRNA aminoacylation process; catalytic activity, oxidoreductase activity and binding were the three main molecular functions of the identified DEGs.

In addition, an obvious positive correlation between the number of enriched DGEs and the concentration of PAs were found in the three biological functions. Especially in the thiamine metabolic process and tRNA aminoacylation, as the concentration of PAs was increased to $1.0 \mathrm{~g} / \mathrm{L}$, more than 6 times of the numbers of DGEs were enriched compared to that in the $0.1 \mathrm{~g} / \mathrm{L}$ PAs-treated group. It indicated that PAs could act as an exogenous regulator to perturb more genes expression to protect yeast cells against the stress factors during fermentation. 


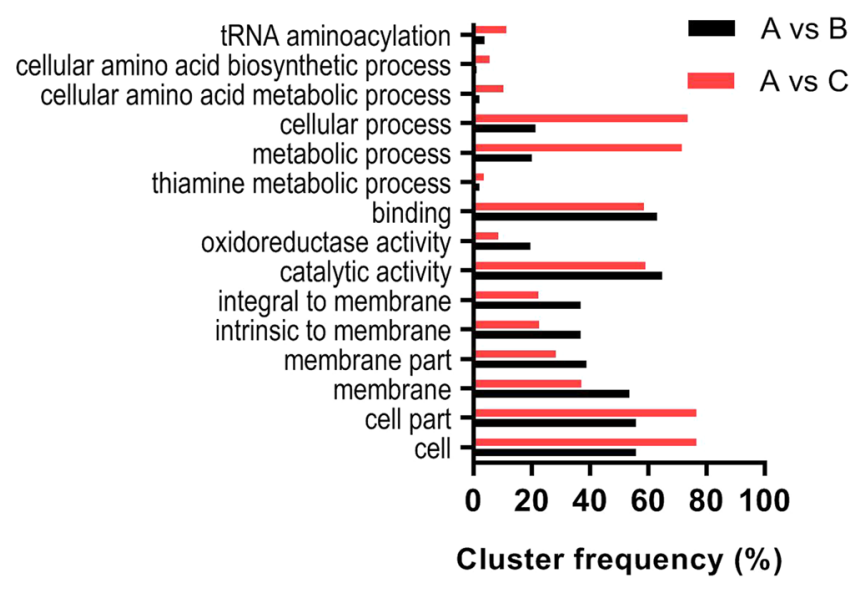

Figure 3. Gene ontology (GO) analysis of the identified DEGs.

Metabolic pathways enrichment analysis. To explore the basic protection mechanism of PAs in yeast cells, the main metabolic or signaling pathways were enriched using the KEGG database (Table 2). In the comparison between PAs treated and untreated groups, six pathways, including vitamin $\mathrm{B}_{6}$ metabolism, thiamine metabolism, biosynthesis of amino acids, aminoacyl-tRNA biosynthesis, starch and sucrose metabolism and steroid biosynthesis were significantly enriched ( $\mathrm{p}$ values were shown in Table 2). Most of the enriched genes within the identified pathways were up-regulated significantly ( $p$ values were shown in Table 2 ), indicating that PAs could be act as an extracellular enhancer to regulate the important metabolic pathways to produce more anabolic products (e.g. vitamins, amino acid, glucan, ergosterol and fatty acid) necessary to survive in wine fermentation process.

Quantitative Real-time PCR. To further confirm the alterations of gene expressions in PAs-treated yeast cells, we measured the relative expression level of four DEGs related to the metabolic pathway of B-complex vitamins $\left(B_{1}, B_{6}\right)$ by RT-qPCR (Fig. 4). The results of RT-qPCR showed a good consistence with that obtained in RNA-seq analysis. The expression levels of the selected genes were up-regulated significantly in PAs-treated groups as relative fold change to the control. More interestingly, a dose-dependent up-regulation appeared as the concentration of PAs increased to $1.0 \mathrm{~g} / \mathrm{L}$. Therefore, the RNA-seq could provide reliable data for differential gene expression analysis.

Based on the results of transcriptome analysis, the genes related to the metabolic pathway of B-complex vitamins $\left(B_{1}, B_{6}\right)$ were up-regulated significantly. In addition, the contents of vitamins $B_{1}$ and vitamins $B_{6}$ were measured to verify the impact of PAs from the aspect of yeast cells phenotype (Fig. 5). Calibration curves of peak area-concentration relationship for each vitamin were prepared using a series of mixed solutions at range from 1.0 to $5.0 \mu \mathrm{g} / \mathrm{mL}$. As shown in Supplementary Fig. S4, it showed a good linear relation with correlation coefficients $\left(\mathrm{R}^{2}\right)$ obtained as 0.9947 and 0.9987 for vitamins $B_{1}$ and $B_{6}$ at the selected concentration ranges, respectively. This method was suitable for the calculation of the intracellular contents of the vitamins $B_{1}$ and $B_{6}$. As shown in Fig. 5, significantly increases of the contents of vitamins $B_{1}$ and vitamins $B_{6}$ were detected in PAs-treated yeast cells. For example, vitamins $B_{1}$ and vitamins $B_{6}$ contents in yeast cells treated with $0.1 \mathrm{~g} / \mathrm{L}$ PAs increased significantly from $23.6 \mu \mathrm{g} / \mathrm{g}$ dry weight and $21.6 \mu \mathrm{g} / \mathrm{g}$ dry weight to $28.9 \mu \mathrm{g} / \mathrm{g}$ dry weight $(\mathrm{p}=0.015)$ and $27.4 \mu \mathrm{g} / \mathrm{g}$ dry weight $(\mathrm{p}=0.001)$, respectively. In addition, like the elevation in gene expression levels, obvious dose-dependent increases of intracellular level of vitamins $B_{1}$ and vitamins $B_{6}$ in the PAs-treated yeast cells were observed, especially for the vitamins $\mathrm{B}_{1}$ contents which showed a $16.6 \%$ increase in the $1.0 \mathrm{~g} / \mathrm{L}$ PAs-treated yeast cells compared to the $0.1 \mathrm{~g} / \mathrm{L}$ PAs-treated ones.

To further prove the protection effects of PAs on the yeast cells, measurements of the cell viability were conducted. The yeast cells were cultivated with $1.0 \mathrm{~g} / \mathrm{L}$ PAs, $50 \mathrm{mg} / \mathrm{L}$ vitamin $\mathrm{B}_{1}, 50 \mathrm{mg} / \mathrm{L}$ vitamin $\mathrm{B}_{6}$ and a $1: 1(\mathrm{w} / \mathrm{w})$ mixture of the two vitamins, respectively in MSM medium at $28^{\circ} \mathrm{C}$ for $72 \mathrm{~h}$, and then the viable cells were counted on the MSM agar plates. As shown in Fig. 6, cell survival decreased significantly in the negative control group in which no PAs or vitamins were supplemented. As expected, the viable cells on plates containing PAs, vitamin $B_{1}$, vitamin $\mathrm{B}_{6}$ and mixture of vitamin $\mathrm{B}_{1}$ and $\mathrm{B}_{6}$, respectively, were higher than the control group. Combined with the results in Figs. 4 and 5, we could clearly notice that exogenous PAs could increase the bio-synthetic yields of vitamin $B_{1}$ and vitamin $B_{6}$ to relieve the environmental stresses in yeast cells during red wine fermentation.

It has been reported that yeast membrane fatty acid composition and membrane fluidity were linked to the stress tolerance of the cells. Exogenous ergosterol has been proved to be effective for the enhancement of the tolerance of monoterpenes in S. cerevisiae strain by regulating the fatty acid composition ${ }^{27}$. The intracellular contents of vitamin $B_{1}$ and vitamin $B_{6}$ could pose significant effects on the lipid metabolism. To testify whether the PAs show the similar effects on alternation of lipid metabolism and yeast tolerance for environmental stresses, we studied the changes in fatty acid profile in the yeast cells with or without the treatment of PAs. As shown in Fig. 7, lipid profile of PAs-treated cells revealed a 5.36\% increase in cellular unsaturated fatty acids (C16:1 and C18:1) with respect to cells grown in the absence of PAs. This comprised a 2.93\% increase in C16:1 and a 2.43\% increase in C18:1. Meanwhile, the saturated fatty acids (C16:0 and C18:0) in the PAs-treated cells decreased significantly from $25.4 \%$ to $19.6 \%$ of the total phospholipid fatty acid compared to that in non PAs-treated cells. 


\begin{tabular}{|c|c|c|c|}
\hline Gene name & Description & $\begin{array}{l}\text { log2 } \\
\text { Fold } \\
\text { Change }\end{array}$ & $\begin{array}{l}\text { Corrected } \\
\text { p-value }\end{array}$ \\
\hline Vitamin $B_{6}$ metabolism & & & $4.54 \times 10^{-4}$ \\
\hline SNO3 & putative pyridoxal 5'-phosphate synthase & +1.01 & $1.08 \times 10^{-5}$ \\
\hline SNZ2 & GMP synthase activity & +1.46 & $3.15 \times 10^{-40}$ \\
\hline SNZ3 & Pyridoxal-5'-phosphate synthase & +1.48 & $1.34 \times 10^{-28}$ \\
\hline SNO2 & putative pyridoxal 5'-phosphate synthase & +1.75 & $2.67 \times 10^{-40}$ \\
\hline Thiamine metabolism & & & $8.15 \times 10^{-7}$ \\
\hline THI80 & thiamine diphosphokinase activity & +1.23 & $4.34 \times 10^{-20}$ \\
\hline THI21 & phosphomethylpyrimidine kinase activity & +1.54 & $7.4 \times 10^{-18}$ \\
\hline THI5 & $\begin{array}{l}\text { Protein involved in synthesis of the thiamine } \\
\text { precursor HMP }\end{array}$ & +1.08 & $1.39 \times 10^{-4}$ \\
\hline THI6 & thiamine-phosphate diphosphorylase activity & +1.21 & $9.36 \times 10^{-18}$ \\
\hline THI20 & phosphomethylpyrimidine kinase activity & +1.14 & $2.12 \times 10^{-13}$ \\
\hline THI22 & hydroxymethylpyrimidine phosphate kinases & +1.39 & $1.51 \times 10^{-15}$ \\
\hline THI72 & $\begin{array}{l}\text { Transporter of thiamine or related } \\
\text { compound }\end{array}$ & +1.12 & $5.67 \times 10^{-10}$ \\
\hline $\begin{array}{l}\text { Arginine and proline } \\
\text { metabolism }\end{array}$ & & & $6.43 \times 10^{-4}$ \\
\hline CAR1 & arginase & -2.10 & $2.28 \times 10^{-32}$ \\
\hline CAR2 & L-ornithine transaminase & +1.04 & $4.91 \times 10^{-8}$ \\
\hline PUT2 & 8-1-pyrroline-5-carboxylate dehydrogenase & -1.00 & $1.44 \times 10^{-10}$ \\
\hline PUT4 & Proline permease & -5.40 & $1.72 \times 10^{-147}$ \\
\hline PRO2 & $\gamma$-glutamyl phosphate reductase & -1.26 & $3.12 \times 10^{-17}$ \\
\hline GAP1 & Amino acid permease & -2.09 & $3.23 \times 10^{-18}$ \\
\hline SPE1 & Ornithine decarboxylase & -1.23 & $3.73 \times 10^{-15}$ \\
\hline Aminoacyl-tRNA biosynthesis & & & $5.09 \times 10^{-4}$ \\
\hline MSE1 & glutamate-tRNA ligase activity & +1.84 & $5.04 \times 10^{-50}$ \\
\hline MSW1 & tryptophan-tRNA ligase activity & +1.38 & $1.34 \times 10^{-32}$ \\
\hline MSM1 & methionine-tRNA ligase activity & +1.69 & $6.71 \times 10^{-32}$ \\
\hline MSF1 & phenylalanine-tRNA ligase activity & +1.60 & $1.76 \times 10^{-30}$ \\
\hline MSD1 & aminoacyl-tRNA ligase activity & +1.34 & $9.97 \times 10^{-28}$ \\
\hline AIM10 & proline-tRNA ligase activity & +1.42 & $3.10 \times 10^{-25}$ \\
\hline HER2 & glutaminyl-tRNA synthase activity & +1.12 & $3.00 \times 10^{-17}$ \\
\hline DIA4 & serine-tRNA ligase activity & +1.56 & $8.10 \times 10^{-17}$ \\
\hline TYS1 & tyrosine-tRNA ligase activity & -1.42 & $8.10 \times 10^{-17}$ \\
\hline KRS1 & lysine-tRNA ligase activity & -1.34 & $5.02 \times 10^{-9}$ \\
\hline CDC60 & leucine-tRNA ligase activity & -1.42 & $5.11 \times 10^{-9}$ \\
\hline THS1 & threonine-tRNA ligase activity & -1.20 & $6.05 \times 10^{-9}$ \\
\hline DPS1 & aspartate-tRNA ligase activity & -1.15 & $1.05 \times 10^{-8}$ \\
\hline DED81 & asparagine-tRNA ligase activity & -1.20 & $1.17 \times 10^{-8}$ \\
\hline ALA1 & alanine-tRNA ligase activity & -1.19 & $6.36 \times 10^{-8}$ \\
\hline FRS1 & phenylalanine-tRNA ligase activity & -1.01 & $4.96 \times 10^{-7}$ \\
\hline GLN4 & glutamine-tRNA ligase activity & -1.03 & $9.35 \times 10^{-5}$ \\
\hline Starch and sucrose metabolism & & & $4.56 \times 10^{-8}$ \\
\hline GSC2 & 1,3-beta-D-glucan synthase activity & +1.89 & $1.43 \times 10^{-9}$ \\
\hline DSE4 & glucan endo-1,4-beta-glucanase activity & +1.36 & $8.55 \times 10^{-21}$ \\
\hline Steroid biosynthesis & & & $1.48 \times 10^{-3}$ \\
\hline ERG26 & C-3 sterol dehydrogenase & +1.14 & $1.55 \times 10^{-11}$ \\
\hline ERG5 & oxidoreductase activity & +1.26 & $7.52 \times 10^{-8}$ \\
\hline ERG3 & oxidoreductase activity & +1.25 & $2.11 \times 10^{-7}$ \\
\hline ERG4 & oxidoreductase activity & +2.13 & $6.11 \times 10^{-23}$ \\
\hline ERG28 & ergosterol biosynthesis & +1.77 & $2.81 \times 10^{-10}$ \\
\hline
\end{tabular}

Table 2. The KEGG pathway enrichment analysis. The column of "log2 Fold Change" represents the base-two logarithm value of the fold change of the gene expression; "+" means up-regulated genes; "-" means downregulated genes. Hypergeometric test was used for statistical analysis. The p-values have been corrected for multiple testing by the Benjamini and Hochberg adjustment method. The corrected $\mathrm{p}$ value $<0.05$ plus the absolute $\log 2$ Fold Change value $>1$ were considered statistically significant. 


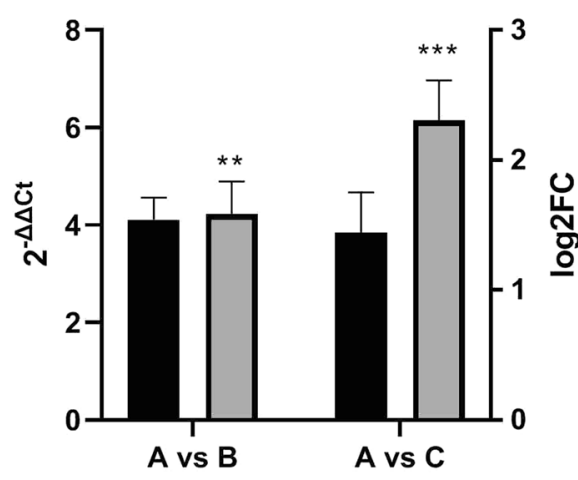

THI80

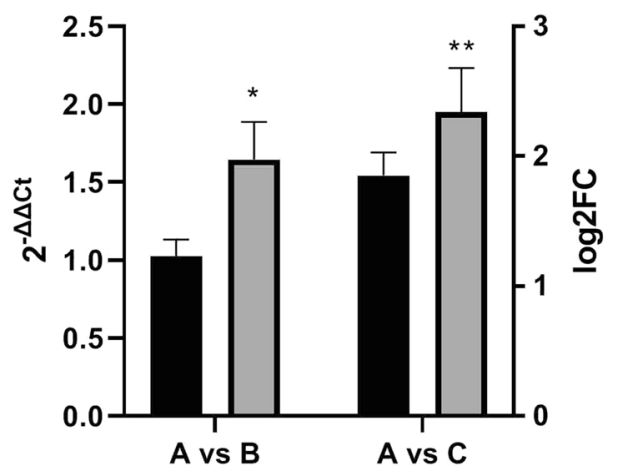

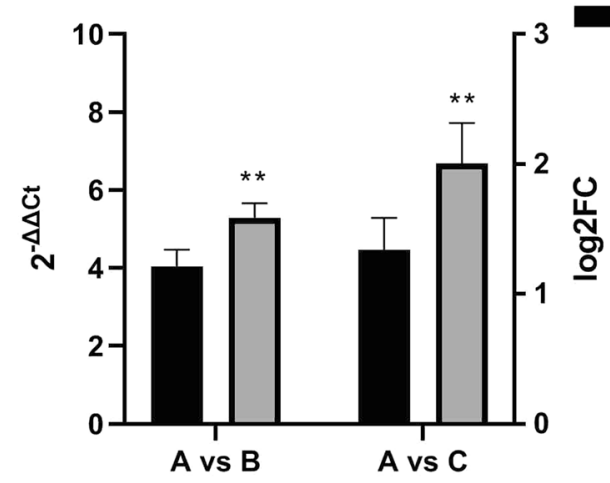

SNZ2

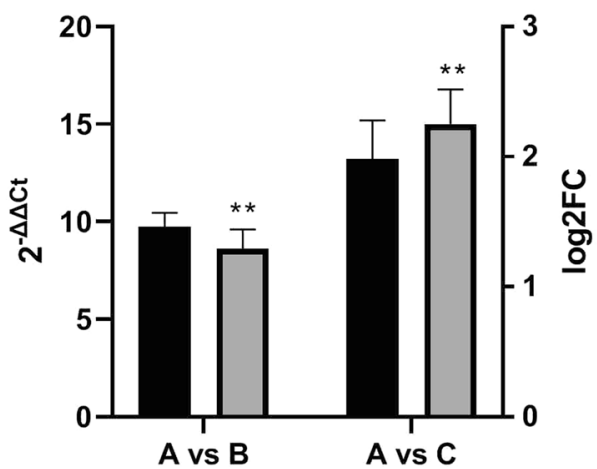

Figure 4. RT-qPCR validations of the results obtained in RNA-seq analysis. The relative expression level of 4 selected genes compared to the actin gene $A C T 1$ was calculated. Value of the $2^{-\Delta \Delta C T}$ was calculated and two-way ANOVA with Tukey's multiple comparisons test were used for the significant analysis of the RT$\mathrm{qPCR}$ at $* \mathrm{p}<0.05, * * \mathrm{p}<0.01, * * * \mathrm{p}<0.001$ level. The absolute $\log 2$ Fold Change value $>1$ were considered statistically significant in the RNA-seq analysis. Three biological replicates were prepared and the data were shown as means \pm S.D.

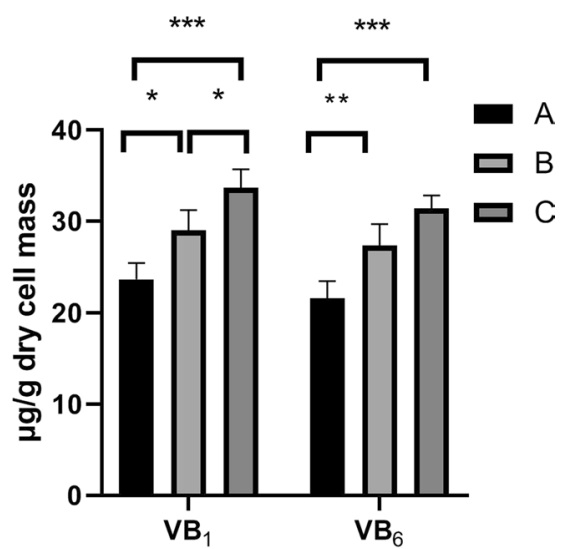

Figure 5. Intracellular contents of vitamins $B_{1}$ and vitamins $B_{6}$ in different systems. All data represent the mean values \pm S.D. from three independent experiments. Two-way ANOVA with Tukey's multiple comparisons test were used for the significant analysis at $* \mathrm{p}<0.05, * * \mathrm{p}<0.01, * * * \mathrm{p}<0.001$ level.

\section{Discussion}

Generally speaking, red wine fermentation process could be considered as the interaction of yeast cells and the complex composition of the grape, especially the sugar, organic acid and phenolic compounds ${ }^{28}$. Sensory attributes of wine including color intensity and stability, mouth-feel, astringency and taste mainly come from the phenolic compounds evolution, especially PAs, the principal constitute in the phenolic compounds ${ }^{29}$. In order to yield high quality red wine with improved sensory and biological properties, thoroughly studies of interactions 


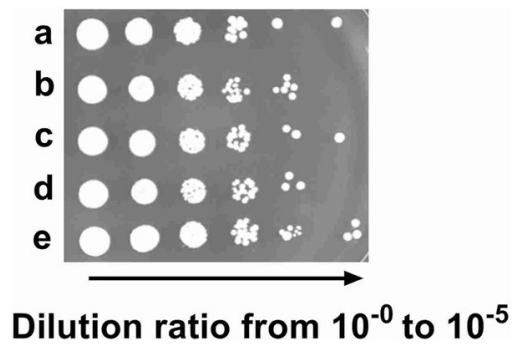

Figure 6. Spot assay of the viability of yeast cells treated with $1.0 \mathrm{~g} / \mathrm{L}$ PAs (b), $50 \mathrm{mg} / \mathrm{L}$ vitamin $\mathrm{B}_{1}$ (c), $50 \mathrm{mg} / \mathrm{L}$ vitamin $B_{6}(d)$ and a 1:1(w/w) mixture of the two vitamins (e), respectively in MSM medium. The negative control (a) was set without the above supplements. After cultivation at $28^{\circ} \mathrm{C}$ for $72 \mathrm{~h}$, five microliter of each proper dilution was spotted on the MSM agar plate for viable cell counts. The arrow below the panel indicates a serial dilution of plated cells $\left(1: 1 ; 1: 10 ; 1: 10^{2} ; 1: 10^{3} ; 1: 10^{4 ;} 1: 10^{5}\right.$ from left to right).

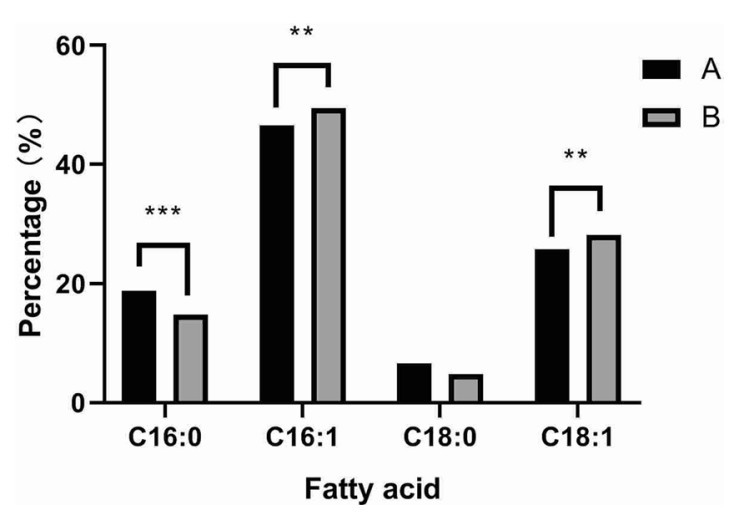

Figure 7. Fatty acid profiles in the membrane of yeast cells. S. cerevisiae yeast strains AWRI R2 were cultivated statically at $28^{\circ} \mathrm{C}$ for $72 \mathrm{~h}$ with (grey bars) or without (dark bars) the treatment of PAs. And then membrane fatty acid compositions were measured. Data were shown as the mean \pm S.D. of three independent experiments. Two-way ANOVA with Tukey's multiple comparisons test were used for the significant analysis at *p $<0.05$, $* * \mathrm{p}<0.01, * * * \mathrm{p}<0.001$ level.

between yeast cells and PAs should be done. In our previous studies, we have showed that the concentration and structure of PAs evolved progressively in a strain- and time-dependent manner during fermentation ${ }^{1}$. Moreover, PAs could pose positive effects on the yeast cells that have proved from the aspects of glucose transport, the energy and redox homeostasis as well as the activities of rate-limiting enzymes in glycolysis ${ }^{24}$. However, to the best of our knowledge, the global regulation posed by PAs from the genetic and metabolic level has never been reported before.

With the rapid development of sequencing technology, RNA-seq has become more efficient and cost-effective in recent years ${ }^{26}$. Identifying key genes and metabolic pathways related to the stress-tolerance abilities of $S$. cerevisiae has been considered as the basis of understanding the intracellular global regulation patterns and making the wine fermentation process more controllable ${ }^{30}$. Therefore, in the present study, we employed the RNA-seq as well as bioinformatics analysis to uncover the possible protection mechanism of PAs for S. cerevisiae during wine fermentation.

We identified a great many of DEGs by measuring the expression levels in the yeast cells cultivated with $0 \mathrm{~g} / \mathrm{L}$ (group A), $0.1 \mathrm{~g} / \mathrm{L}$ (group B), 1.0 g/L (group C) PAs, respectively. By GO and KEGG enrichment analysis, the gene functions and participated metabolic pathways have been uncovered. The significantly enriched gene functions were about the structure of cell membrane, thiamine metabolic processes, cellular amino acid metabolic processes, tRNA amino acylation process, oxidoreductase activity and binding activity. The identified DEGs were significantly enriched in to six metabolic pathways including vitamin $\mathrm{B}_{6}$, thiamine, amino acids, aminoacyl-tRNA, carbohydrate and steroid. Most of the enriched genes in the metabolic pathways of vitamin $\mathrm{B}_{6}$, thiamine, starch and sucrose and steroid showed significantly up-regulated expression patterns compared to that of control group, indicating PAs could act as an extracellular activator to enhance the metabolic efficiency of vitamin $\mathrm{B}_{6}$, thiamine, carbohydrates and steroids.

In our study, for the first time we showed that the genes (SNO3, SNZ2, SNZ3, SNO2, THI80, THI21, THI6, THI20, THI72, THI5, THI22) in the PAs-treated yeast cells performed significantly higher expression levels, involving in the sequentially enhanced bio-synthesis of vitamin $B_{1}$ and $B_{6}$ for yeasts (Figs. 4 and 5). It has been reported that increased amplification number of these genes related to bio-synthesis of vitamin $B_{1}$ and vitamin $B_{6}$ confers the efficient growth of yeast cells in the thiamin and/or pyridoxine-deficient medium with high sugar concentrations ${ }^{31}$. Interestingly, our results consistent with the previous study that the yeast cells 
supplemented with PAs and vitamin $\mathrm{B}_{1}$ and $\mathrm{B}_{6}$ compounds showed higher viability compared to the control group (Fig. 6). It deduced there must be problems with the bio-availability of these vitamins in the wine making process and exogenous PAs could cover the deficiency of the two important vitamins for yeast cells against environmental stresses ${ }^{32}$. It also has reported that decreased intracellular vitamin $\mathrm{B}_{6}$ content could repress many metabolic pathways, including reduced respiration activity and a significant alteration in sterol and unsaturated fatty acids metabolism ${ }^{33}$. It might resulted in the sequential changes in the composition of lipids, affecting the fluidity and permeability of cell membrane, as wells as the cell growth ${ }^{27}$. In our study, we also studied the fatty acid profiles in the membrane of yeast cells with or without the treatment of PAs. A significant increase in the quantities of unsaturated fatty acids (C16:1 and C18:1) was found in the presence of PAs compared to the control group, whereas the quantities of palmitic acid (C16:0) and stearic acid (C18:0) decreased significantly, reflecting that an indirect interaction between PAs and the metabolisms of fatty acid and vitamin $B_{6}$ might exist in yeast cells by which the phenolic compounds in wine could enhance the physiological activities and fermentation performance. Higher contents of unsaturated fatty acids in yeast cells membranes could make the yeast cells less sensitive to heat stress by repressing the expression level of genes involving the heat-shock response (HSR) and general stress response (GSR) pathways ${ }^{34}$. In other words, there was an obvious positive correlation between the contents of unsaturated fatty acids and stress tolerance capacity of yeast strains. Our findings provided an exciting possibility that it might be possible to make yeast cells more robust by simply modifying the percentage of PAs in the medium.

Thiamine is another essential molecule for all living organisms. Deficiency in thiamine could decrease NADPH, which affected cellular anabolism and lead to a cessation of glucose consumption ${ }^{35}$. As a cofactor of transketolase in pentose phosphate pathway, thiamine also could accelerate the metabolism from fructose 6-phosphate and glyceraldehyde 3-phosphate to ribulose-5-phosphate and erythritol 4-phosphate, precursors for the synthesis of amino acids in yeast cells under stress situations ${ }^{36}$. Our previous study showed that PAs could pose significant enhancement on the activity of $\mathrm{H}^{+}$-ATPase, cell growth, and alcoholic fermentation rate by up-regulating the gene expression levels of PMA1 and HXT7, encoding the plasma membrane ATPase and yeast hexose transporter, respectively $y^{24,25}$. That is, the benefits provided by exogenous PAs to the growth and fermentation performances of yeast cells could be explained from the interaction between the phenolic substances in wine and the metabolisms of thiamine and amino acid.

In our study, the bio-synthesis pathway of ergosterol was also significantly enriched that the expression of five related genes (eg. ERG3, ERG4, ERG5, ERG26, ERG28) were highly induced in the yeast cells with the treatment of PAs. Ergosterols are essential structural and regulatory components involving in the fluidity and permeability of yeast cells membrane. Higher contents of ergosterol could protect yeast against multiple environmental stresses such as oxidative, ethanol, osmotic and so on ${ }^{37}$. Enhanced amplification numbers of ergosterol synthesis-related genes in yeast cells have been proved to be connected with the PAs supplementation (Table 2). Exogenous PAs might affect the contents of ergosterol and in sequentially contribute for the enhancement of stress tolerance ability in the yeast cells which should be further verified in the future.

It has been reported accumulation of amino acids in plant or microorganisms was critical for the environmental stresses tolerance. The accumulated amino acids could act as osmoprotectants, ROS scavenger and protector against heavy metals $s^{38-40}$. The possibility is that free amino acid could interfere with the side chain bonding and introduce conformational changes in the enzyme protein and thus affect the enzymes synthesis and activity ${ }^{41}$. Our data demonstrated the pathways of arginine and proline metabolism and aminoacyl-tRNA biosynthesis were significantly enriched in PAs-supplemented yeast cells by the KEGG analysis. Arginine and proline were considered as the important osmoprotectant, playing pivotal role of metabolism in cellular osmoregulation $^{42}$. The expression level of genes involving in the arginine and proline metabolism showed up or down-regulated patterns (Table 2). CAR2 gene expression level was up-regulated significantly by feeding with PAs, and it might contribute to more synthesis of L-ornithine transaminase which could catalyze glutamate- $\gamma$-semialdehyde (GSA) to ornithine in cytosol, the precursor of arginine, more efficiently and alleviate the encountered environmental stress ${ }^{43}$. The uptake for proline in yeast was largely mediated by a high affinity, specific permease, Put4p, and a low affinity general amino acid permease, Gaplp, encoded by PUT4 and GAP1, respectively ${ }^{44}$. However, in our study, the gene expression level of PUT4 and GAP1 in the PAs-treated yeast cells showed significant down-regulated. The two inconsistent metabolic patterns of arginine and proline might due to the species-specific alternation or the PAs regulation which should be further studied. Other down-regulated genes (CAR1, SPE1, and PUT2) repressed the degradation pathways of the two kinds of protective amino acids. The gene $\mathrm{PRO} 2$ (encoding $\gamma$-glutamyl phosphate reductase) were involving in the catalysis of the second step in proline biosynthesis from glutamate. Glutamate and proline were both important amino acid in protecting yeast cells against stress factors. Pyrroline-5-carboxylate (P5C) was an critical reaction intermediate making it possible of the interconversion of glutamate, ornithine, proline and arginine ${ }^{45}$. In PAs-treated yeast cells the gene expression level of PRO2 was down-regulated significantly, reflecting the cells decreased the consumption of glutamate for the accumulation of proline. It might deduce the phenolic compounds could act as a regulator for the dynamic balance of the intracellular amino acids in yeast cells during wine fermentation.

During wine fermentation, a global regulation is required to maintain the intracellular homeostasis for the survival and growth of the yeast cells. All of the physiological activities could be considered as the enzymatic reactions. Therefore, it was vital that translation reprogramming should be in a correct and controllable way ${ }^{46}$. In our study, a large amount of genes involving in the aminoacyl-tRNA biosynthesis were differential expressed significantly in PAs-treated yeast cells during fermentation. It indicated that the phenolic compounds in wine could regulate the biosynthesis and activity of aminoacyl-tRNA ligase, and subsequently affect the protein translation globally as the cells responded to the environmental stresses to maintain the intracellular homeostasis. 


\section{Conclusions}

RNA-seq analysis was employed in our study to systematically explore the protection mechanism of PAs on the yeast cells during wine fermentation. A large amount of DEGs were identified and subsequently enriched into six metabolic pathways including vitamin $\mathrm{B}_{6}$, thiamine, amino acids, aminoacyl-tRNA, carbohydrate and steroid. The intracellular contents of the thiamine and vitamin $\mathrm{B}_{6}$ increased significantly in PAs-treated yeast cells, which was in consistent with the cell viability and the expression pattern of the identified DEGs participating in the metabolic pathways of vitamin $B_{6}$ and thiamine. In addition, it revealed a significant difference in the fatty acid composition between the PAs-treated yeast cells and the control that the UFA (C16:1 and C18:1) increased whereas SFA (C16:0 and C18:0) decreased. The other enriched metabolic pathways in RNA-seq analysis such as the biosynthesis of amino acids, proteins and steroids should be mainly focused on in further study to fully elucidate the interactions between the phenolic compounds in wine and the yeast cells. In general, data of this study provided insights into the possible molecular mechanisms of PAs protection in S. cerevisiae yeast strains during wine fermentation process and might contribute to the selection and modification of yeast strains with enhanced resistance capacity.

\section{Materials and methods}

Yeast strain and culture conditions. AWRI R2, used in this study, was a commercial yeast strain from Marivin (Queensland, Australian) and has good fermentation performance. Yeast cells were maintained on YPD medium slants ( $1 \%$ yeast extract, $2 \%$ peptone, $2 \%$ glucose, $2 \%$ agar). Fresh yeast inoculum was prepared by transferring one loop of yeast colony into $100 \mathrm{~mL}$ YPD medium in a $250 \mathrm{~mL}$ flask and incubating aerobically overnight in a shaker at $28^{\circ} \mathrm{C}$ and $120 \mathrm{rpm}$.

Fermentation. Model synthetic medium (MSM) was used for fermentation in this study. The MSM is a chemically well-defined medium to simulate a standard grape juice for wine fermentation in laboratory ${ }^{47}$. The composition of this medium was as follows: glucose ( $100 \mathrm{~g})$, fructose $(100 \mathrm{~g})$, tartaric acid ( $3 \mathrm{~g})$, citric acid (0.3 g), L-malic acid (0.3 g), $\mathrm{MgSO}_{4}(0.2 \mathrm{~g}), \mathrm{KH}_{2} \mathrm{PO}_{4}(2 \mathrm{~g}),(\mathrm{NH} 4)_{2} \mathrm{SO}_{4}(0.3 \mathrm{~g})$, asparagine $(0.6 \mathrm{~g}), \mathrm{MnSO}_{4} \cdot \mathrm{H}_{2} \mathrm{O}(4 \mathrm{mg})$, $\mathrm{ZnSO}_{4} \cdot 7 \mathrm{H}_{2} \mathrm{O}(4 \mathrm{mg}), \mathrm{CuSO}_{4} \cdot 5 \mathrm{H}_{2} \mathrm{O}(1 \mathrm{mg}), \mathrm{KI}(1 \mathrm{mg}), \mathrm{CoCl}_{2} \cdot 6 \mathrm{H}_{2} \mathrm{O}(0.4 \mathrm{mg}),\left(\mathrm{NH}_{4}\right)_{6} \mathrm{Mo}_{7} \mathrm{O}_{24} \cdot 4 \mathrm{H}_{2} \mathrm{O}(1 \mathrm{mg}), \mathrm{H}_{3} \mathrm{BO}_{3}$ $(1 \mathrm{mg})$, meso-inositol $(300 \mathrm{mg})$, biotin $(0.04 \mathrm{mg})$, thiamin $(1 \mathrm{mg})$, pyridoxine $(1 \mathrm{mg})$, nicotinic acid (1 mg), pantothenic acid (1 mg), p-amino benzoic acid $(1 \mathrm{mg})$, palmitic acid $(1 \mathrm{mg})$, palmitoleic acid $(0.2 \mathrm{mg})$, stearic acid $(3 \mathrm{mg})$, oleic acid $(0.5 \mathrm{mg})$, linoleic acid $(0.5 \mathrm{mg})$ and linolenic acid $(0.2 \mathrm{mg})$. The MSM was prepared by mixing thoroughly the above components in $1 \mathrm{~L}$ deionized water and the final $\mathrm{pH}$ value of the medium was adjusted to 3.3. The crude PAs was firstly subjected to a column-based purification procedure according to our previous research protocol ${ }^{25}$ to remove the monomers and oligomers. The purified PAs was weighted accurately and dissolved in $10 \mathrm{~mL}$ absolute ethyl alcohol thoroughly before use. The obtained PAs solutions were added into the MSM medium to make a series of final concentrations of $0.1 \mathrm{~g} / \mathrm{L}, 1.0 \mathrm{~g} / \mathrm{L}$ (marked as group B and group C, respectively). The control group marked as group A was prepared by adding $10 \mathrm{~mL}$ absolute ethyl alcohol without PAs into the fermentation medium directly. The prepared culture media were sterilized by filtration (nitrate cellulose membrane, $0.45 \mu \mathrm{m})$ and supplemented with sulfur dioxide $(20 \mathrm{mg} / \mathrm{L})$ to simulate the oenological environment before yeast strain inoculation.

For the fermentation, one percent of the prepared yeast inoculum was inoculated into $400 \mathrm{~mL}$ MSM medium supplemented with $0,0.1$ and $1.0 \mathrm{~g} / \mathrm{L}$ PAs in $500 \mathrm{~mL}$ flasks equipped with glass capillary stoppers to maintain the anaerobic conditions and cultivated statically at $28^{\circ} \mathrm{C}$. Samples were taken at the $72 \mathrm{~h}$ that the yeast cells were staying in the stationary phase. Ten milliliter of the fermentation broth was centrifuged at $6000 \mathrm{r} / \mathrm{min}$ for 5 min to collect the yeast cell pellets. The obtained yeast cells were washed 3 times with $0.9 \%$ sodium chloride solution and rapidly frozen in liquid nitrogen before stored at $-80^{\circ} \mathrm{C}$ for the following experiments.

RNA extraction and sequencing. Yeast cells were grinded in liquid nitrogen. Total RNA of each sample was extracted using RNeasy Mini Kit (Qiagen, Germany). The obtained RNA products were quantified and qualified by Agilent 2100 Bioanalyzer (Agilent Technologies, Palo Alto, CA, USA), NanoDrop (Thermo Fisher Scientific Inc.) and $1 \%$ agarose gel electrophoresis before next generation sequencing library preparations. One microgram of total RNA passing the quality test was used for library preparation based on the manufacturer's protocol of the NEBNext Ultra RNA Library Prep kit for Illumina.

Illumina HiSeq X10 sequencing platform was employed for the library sequencing using a $2 \times 150 \mathrm{bp}$ paired-end (PE) configuration; image analysis and base calling were conducted by the HiSeq Control Software (HCS) + OLB + GAPipeline-1.6 (Illumina) on the HiSeq instrument. The sequences were processed and analyzed by Genewiz (Suzhou, China).

Differential gene expression analysis. Quality control for the sequencing reads were conducted by removing low quality reads containing adapter, primers and poly- $\mathrm{N}$ to generate the clean data by Trimmomatic (v0.30). The reference genome sequences and gene model annotation files of S. cerevisiae S288C were downloaded from NCBI https://www.ncbi.nlm.nih.gov/genome/genomes/15 for the reads mapping. Paired-end clean reads were aligned to the reference genome via Hisat2 software (v2.0.1). RPKM (reads per kilo bases per million reads) values were calculated as the indicators of gene expression abundance. DESeq Bioconductor package, a model based on the negative binomial distribution, was used for the differential expression analysis. After adjusted by Benjamini and Hochberg's approach for controlling the false discovery rate, $p$-value $<0.05$ and the absolute value of $\log 2$ fold change $>1$ were set together to detect differential expressed ones ${ }^{48}$.

Enrichment analysis. GO-TermFinder was used for identifying Gene Ontology (GO) terms that annotate a list of enriched genes with a significant p-value $<0.05$. To identify the key metabolic pathways, KOBAS software 2.0 was employed to enrich significant differential expression gene in KEGG pathways. 


\begin{tabular}{|l|l|l|l|l|}
\hline $\begin{array}{l}\text { Gene } \\
\text { name }\end{array}$ & Gene description & Forward primer $\left(5^{\prime}-\mathbf{3}^{\prime}\right)$ & Reverse primer $\left(5^{\prime}-\mathbf{3}^{\prime}\right)$ & $\begin{array}{l}\text { Product } \\
\mathbf{( b p})\end{array}$ \\
\hline SNZ2 & pyridoxal $5^{\prime}$-phosphate synthase & CAAGGATCTAGGTGAGGCTTTG & CTGGATCTCCGCCTTAATCTTG & 130 \\
\hline THI21 & hydroxymethylpyrimidine kinase & ATTTGGCTCGCGGTTATTCC & GCCCGTTGTCCTTAACAGTC & 114 \\
\hline THI6 & thiamine-phosphate diphosphorylase & TATGGCAATTGACGCCGATG & GCCAATGTCTCTACCTCCGA & 132 \\
\hline THI80 & Thiamine pyrophosphokinase & ATCCATCCAAACGAGGATGA & CTATTGGCTGCACCATCTGC & 131 \\
\hline ACT1 & Actin & ACATCGTTATGTCCGGTGGT & CCACCAATCCAGACGGAGTA & 142 \\
\hline
\end{tabular}

Table 3. Primer lists used in RT-qPCR reactions.

RT-qPCR validation. Four candidates of the differentially expressed genes associated with the vitamin B complex biosynthesis pathway were selected to validate the transcriptome data using quantitative real-time PCR (RT-qPCR) and the list of the genes and primers were shown in Table 3. Amplification and detection were undertaken with a Thermal Cycler Dice Real-Time System (Takara Bio., Kyoto, Japan). The reaction mixtures were consisted with $12.5 \mu \mathrm{L}$ of SYBR Premix Ex Taq II (Takara Bio.), $0.5 \mu \mathrm{L}$ of each primer $(10 \mu \mathrm{M})$ and $2 \mu \mathrm{L}$ of prepared cDNA made up to a total volume of $25 \mu \mathrm{L}$. Forty cycles at $95^{\circ} \mathrm{C}$ for $10 \mathrm{~s}$ and at $55^{\circ} \mathrm{C}$ for $10 \mathrm{~s}$ and at $72^{\circ} \mathrm{C}$ for $10 \mathrm{~s}$ after the primary denaturation at $95^{\circ} \mathrm{C}$ for $10 \mathrm{~s}$ were run for the PCR program. The $\beta$-Actin gene ACT1 was used as an internal gene expression control. The whole experiment was repeated for three times. The value of $2^{-\Delta \Delta C T}$ was calculated and used as the indicator for the relative expression level of the target genes ${ }^{49}$.

Measurements of $B$-complex vitamins $\left(B_{1}, B_{6}\right)$. In order to verify the up-regulated genes participating into the metabolic pathway of vitamin $B_{1}$ and $B_{6}$ from the aspect of yeast phenotype, the intracellular contents of the two vitamins were measured by using reverse phase high performance liquid chromatographic (RP-HPLC) method ${ }^{50}$. The prepared yeast cells were re-suspend in $2 \mathrm{~mL}$ deionized water and almost complete cell breakage was obtained using a mechanical bead beater (Biospec Products) set at $6000 \mathrm{rpm}$ for four $30 \mathrm{~s}$ periods alternating with $30 \mathrm{~s}$ intervals on ice bath. Supernatant were collected by centrifugation at $10,000 \mathrm{rpm}$ for $2 \mathrm{~min}$ for the chromatographic measurement. About $50 \mathrm{mg}$ each of thiamine hydrochloride $\left(\mathrm{VB}_{1}\right)$ and pyridoxine hydrochloride $\left(\mathrm{VB}_{6}\right)$ standard chemicals was weighed accurately and dissolved in $50 \mathrm{~mL} \mathrm{1:1} \mathrm{(v/v)} \mathrm{distilled} \mathrm{water/} \mathrm{acetonitrile} \mathrm{solution} \mathrm{thoroughly} \mathrm{for} \mathrm{the} \mathrm{prepara-}$ tion of mixed stock standard solutions. The stock standard solutions were filtered through $0.45 \mu \mathrm{m}$ membrane filter and diluted with distilled water in the test tubes to yield five series of mixed standard solutions.

The separations and quantitative determination were performed on a Waters 2996 HPLC system (Waters Corp., Milford, MA, USA) consisting of an C18 reverse phase column (Waters X-Terra RP $18250 \times 4.6 \mathrm{~mm}$ id., $5 \mu \mathrm{m}$ particle size), a guard column containing the same material and Waters series 2487 spectrophotometric detector. The wavelength was set at $320 \mathrm{~nm}$ to separate all the two metabolites in a single injection. The degassed binary mobile phase consisted of $30 \%$ ortho phosphoric acid (Solvent A) and $70 \%$ acetonitrile (Solvent B). The flow rate was $1.2 \mathrm{~mL} / \mathrm{min}$ and the volume injected was $20 \mu \mathrm{L}$. Sample peaks were identified by comparing the retention times with those of the standard chemicals. Integration of separated peak area was calculated and the content of each vitamin was determined by using the calibration curve of peak area-concentration relationship. The vitamin contents ( $\mu \mathrm{g} / \mathrm{g}$ dry cell weight) in each sample were expressed as the normalized values by dry cell mass weight.

Measurement of cellular fatty acid composition. Freeze dried yeast cells were weighted and subjected to the lipid extraction according to the literature protocol with slightly modification ${ }^{51}$. Briefly, the cells were disrupted in a MiniBeadbeater-16 (Biospec Products Inc., Bartlesville, OK, USA) according to our previous study ${ }^{52}$. Lipids were extracted from the cell homogenates with the mixed solution of chloroform and methanol (2:1, v/v). Fatty acid methyl esters (FAMEs) were prepared by catalytic reaction of $2 \% \mathrm{H}_{2} \mathrm{SO}_{4}$ in anhydrous methanol at $70^{\circ} \mathrm{C}$ for $2 \mathrm{~h}$ and then the FAMEs solutions were analyzed by gas chromatography (Shimadzu GC-2010 plus), with a QP-5000 mass spectrometer, on an Agilent DB-17 capillary column (30 m length, $0.25 \mathrm{~mm}$ inner diameter, $0.25 \mu \mathrm{m}$ film thickness). FAMEs were identified by comparing the corresponding mass spectra with a spectrum database. The fatty acid composition of each sample was determined based on the integrated peak area for each FAMEs shown as a percentage of the total peak area.

Spot assay. In order to verify the physiological functions of the increased intracellular contents of vitamin $B_{1}$ and $\mathrm{B}_{6}$ as well as the up-regulated expression level of the encoding genes, spot assay of the viable cell counts were conducted. The harvested fresh cells were re-suspended in the MSM medium supplemented with $1.0 \mathrm{~g} / \mathrm{L} \mathrm{PAs,} 50 \mathrm{mg} / \mathrm{L}$ vitamin $\mathrm{B}_{1}$, $50 \mathrm{mg} / \mathrm{L}$ vitamin $B_{6}$ and a 1:1(w/w) mixture of the two vitamins, respectively. The negative control without the above supplements was set for the comparison of cell viability. All the samples were cultivated statically at $28^{\circ} \mathrm{C}$ for $72 \mathrm{~h}$ and then serially diluted. Five microliter of proper dilutions was spotted on the MSM agar plate for viable cell counts.

Received: 19 December 2019; Accepted: 31 March 2020;

Published online: 21 April 2020

\section{References}

1. Li, J., Zhao, H. \& Huang, W. Yeast-induced changes in the concentration and structure of oligomeric proanthocyanidins during simulated wine fermentation. Aust. J. Grape Wine R. 21, 404-410, https://doi.org/10.1111/ajgw.12140 (2015).

2. Aron, P. M. \& Kennedy, J. A. Flavan-3-ols: Nature, occurrence and biological activity. Mol. Nutr. Food Res. 52, 79-104, https://doi. org/10.1002/mnfr.200700137 (2008). 
3. Ghosh, D. \& Scheepens, A. Vascular action of polyphenols. Mol. Nutr. Food Res. 53, 322-331, https://doi.org/10.1002/ mnfr.200800182 (2009)

4. Serrano, J., Puupponen-Pimiä, R., Dauer, A., Aura, A.-M. \& Saura-Calixto, F. Tannins: Current knowledge of food sources, intake, bioavailability and biological effects. Mol. Nutr. Food Res. 53, S310-S329, https://doi.org/10.1002/mnfr.200900039 (2009).

5. Terra, X. et al. Grape-seed procyanidins prevent low-grade inflammation by modulating cytokine expression in rats fed a high-fat diet. J. Nutr. Biochem. 20, 210-218, https://doi.org/10.1016/j.jnutbio.2008.02.005 (2009).

6. Kennedy, J. A., Matthews, M. A. \& Waterhouse, A. L. Effect of maturity and vine water status on grape skin and wine flavonoids. Am. J. Enol. Viticult. 53, 268-274 (2002).

7. Kovac, V., Alonso, E., Bourzeix, M. \& Revilla, E. Effect of several enological practices on the content of catechins and proanthocyanidins of red wines. J. Agr. Food Chem. 40, 1953-1957 (1992).

8. Scudamore-Smith, P., Hooper, R. \& McLaran, E. Color and phenolic changes of Cabernet Sauvignon wine made by simultaneous yeast/bacterial fermentation and extended pomace contact. Am. J. Enol. Viticult. 41, 57-67 (1990).

9. Recamales, Á. F., Sayago, A., González-Miret, M. L. \& Hernanz, D. The effect of time and storage conditions on the phenolic composition and colour of white wine. Food Res. Int. 39, 220-229 (2006).

10. Vivar-Quintana, A., Santos-Buelga, C., Francia-Aricha, E. \& Rivas-Gonzalo, J. Formation of anthocyanin-derived pigments in experimental red wines. Food Sci. Technol. Int. 5, 347-352 (1999).

11. Mazauric, J.-P. \& Salmon, J.-M. Interactions between yeast lees and wine polyphenols during simulation of wine aging: I. Analysis of remnant polyphenolic compounds in the resulting wines. J. Agr. Food Chem. 53, 5647-5653 (2005).

12. Gawel, R. Red wine astringency: a review. Aust. J. Grape Wine R. 4, 74-95 (1998).

13. Morata, A. et al. Adsorption of anthocyanins by yeast cell walls during the fermentation of red wines. J. Agr. Food Chem. 51, 4084-4088 (2003).

14. Bauer, F. \& Pretorius, I. S. Yeast stress response and fermentation efficiency: how to survive the making of wine-a review. S. Afr. J. Enol. Vitic. 21, 27-51 (2000).

15. Olofsson, K., Bertilsson, M. \& Lidén, G. A short review on SSF-an interesting process option for ethanol production from lignocellulosic feedstocks. Biotechnol. biofuels 1, 7 (2008).

16. Carrasco, P. \& Querol, A. Analysis of the stress resistance of commercial wine yeast strains. Arch. Microbiol. 175, 450-457 (2001).

17. Erasmus, D. J., Cliff, M. \& van Vuuren, H. J. Impact of yeast strain on the production of acetic acid, glycerol, and the sensory attributes of icewine. Am. J. Enol. Viticult. 55, 371-378 (2004).

18. Querol, A., Fernández-Espinar, M. T., ll del Olmo, M. \& Barrio, E. Adaptive evolution of wine yeast. Int. J. Food Microbiol. 86, 3-10 (2003).

19. Zaky, A. S., Greetham, D., Tucker, G. A. \& Du, C. The establishment of a marine focused biorefinery for bioethanol production using seawater and a novel marine yeast strain. Sci. Rep. 8, 1-14 (2018).

20. Cardona, F., Carrasco, P., Pérez-Ortín, J. E., lí del Olmo, M. \& Aranda, A. A novel approach for the improvement of stress resistance in wine yeasts. Int. J. Food Microbiol. 114, 83-91 (2007).

21. Sablayrolles, J. Control of alcoholic fermentation in winemaking: Current situation and prospect. Food Res. Int. 42, 418-424 (2009).

22. Reguant, C., Bordons, A., Arola, L. \& Rozes, N. Influence of phenolic compounds on the physiology of Oenococcus oeni from wine. J. Appl. Microbiol. 88, 1065-1071 (2000).

23. Vivas, N., Lonvaud-Funel, A. \& Glories, Y. Effect of phenolic acids and anthocyanins on growth, viability and malolactic activity of a lactic acid bacterium. Food Microbiol. 14, 291-299 (1997).

24. Li, J., Zhao, H. \& Huang, W. Mechanism of proanthocyanidins-induced alcoholic fermentation enhancement in Saccharomyces cerevisiae. J. Ind. Microbiol. Biot. 41, 1793-1802, https://doi.org/10.1007/s10295-014-1517-1 (2014).

25. Li, J., Du, G., Yang, X. \& Huang, W. Effect of proanthocyanidins on yeast metabolism, H+ -ATPase activity, and wine fermentation. Am. J. Enol. Viticult. 62, 512, https://doi.org/10.5344/ajev.2011.11021 (2011).

26. Metzker, M. L. Sequencing technologies-the next generation. Nat. Rev. Genet. 11, 31-46 (2010).

27. Liu, J., Zhu, Y., Du, G., Zhou, J. \& Chen, J. Exogenous ergosterol protects Saccharomyces cerevisiae from D-limonene stress. J. Appl. Microbiol. 114, 482-491, https://doi.org/10.1111/jam.12046 (2013).

28. Fleet, G. H. Yeast interactions and wine flavour. Int. J. Food Microbiol. 86, 11-22, https://doi.org/10.1016/S0168-1605(03)00245-9 (2003).

29. Soares, S., Brandão, E., Mateus, N. \& de Freitas, V. Sensorial properties of red wine polyphenols: Astringency and bitterness. Crit. Rev. Food Sci. 57, 937-948, https://doi.org/10.1080/10408398.2014.946468 (2017).

30. Walker, M. E. et al. Genome-wide identification of the Fermentome; genes required for successful and timely completion of winelike fermentation by Saccharomyces cerevisiae. BMC Genomics 15, 552, https://doi.org/10.1186/1471-2164-15-552 (2014).

31. Stambuk, B. U., Dunn, B., Alves, S. L., Duval, E. H. \& Sherlock, G. Industrial fuel ethanol yeasts contain adaptive copy number changes in genes involved in vitamin B1 and B6 biosynthesis. Genome Res. 19, 2271-2278, https://doi.org/10.1101/gr.094276.109 (2009).

32. Matxain, J. M., Padro, D., Ristilä, M., Strid, Å. \& Eriksson, L. A. Evidence of high $\bullet \mathrm{OH}$ radical quenching efficiency by vitamin B6. J. Phys. Chem. B 113, 9629-9632, https://doi.org/10.1021/jp903023c (2009).

33. Kamihara, T. \& Nakamura, Regulation of respiration and its related metabolism by vitamin B1 and vitamin B6 in Saccharomyces yeasts in Immobilized biocatalysts Saccharomyces Yeasts Wastewater Treament (ed. Scheper, T.) 35-82 (Springer Verlag Berlin Heidelberg, 1984)

34. Chatterjee, M. T., Khalawan, S. A. \& Curran, B. P. G. Cellular lipid composition influences stress activation of the yeast general stress response element (STRE). Microbiology 146, 877-884, https://doi.org/10.1099/00221287-146-4-877 (2000).

35. Leite, H. P. \& de Lima, L. F. P. Thiamine (Vitamin B1) deficiency in intensive care: Physiology, risk factors, diagnosis, and treatment in Diet and nutrition in critical care (eds. Rajkumar Rajendram, Victor R. Preedy, \& Vinood B. Patel) 959-972 (Springer New York, 2015).

36. Stincone, A. et al. The return of metabolism: Biochemistry and physiology of the pentose phosphate pathway. Biol. Rev. 90, 927-963, https://doi.org/10.1111/brv.12140 (2015).

37. Landolfo, S. et al. Oleic acid and ergosterol supplementation mitigates oxidative stress in wine strains of Saccharomyces cerevisiae. Int. J. Food Microbiol. 141, 229-235, https://doi.org/10.1016/j.ijfoodmicro.2010.05.020 (2010).

38. Tsolmonbaatar, A. et al. Isolation of baker's yeast mutants with proline accumulation that showed enhanced tolerance to bakingassociated stresses. Int. J. Food Microbiol. 238, 233-240, https://doi.org/10.1016/j.ijfoodmicro.2016.09.015 (2016).

39. Nuccio, M. L., Rhodes, D., McNeil, S. D. \& Hanson, A. D. Metabolic engineering of plants for osmotic stress resistance. Curr. Opin. Plant Biol. 2, 128-134 (1999).

40. Matysik, J., Alia, Bhalu, B. \& Mohanty, P. Molecular mechanisms of quenching of reactive oxygen species by proline under stress in plants. Curr. Sci., 525-532 (2002).

41. Hoque, M. A. et al. Exogenous proline and glycinebetaine increase $\mathrm{NaCl}$-induced ascorbate-glutathione cycle enzyme activities, and proline improves salt tolerance more than glycinebetaine in tobacco Bright Yellow-2 suspension-cultured cells. J. Plant Physiol. 164, 1457-1468 (2007).

42. Long, D., Wilkinson, K. L., Taylor, D. K. \& Jiranek, V. Novel wine yeast for improved utilisation of proline during fermentation. Fermentation 4, 10 (2018) 
43. Nishimura, A., Kotani, T., Sasano, Y. \& Takagi, H. An antioxidative mechanism mediated by the yeast N-acetyltransferase Mpr1: oxidative stress-induced arginine synthesis and its physiological role. FEMS Yeast Res. 10, 687-698, https://doi. org/10.1111/j.1567-1364.2010.00650.x (2010).

44. Poole, K. et al. Proline transport and stress tolerance of ammonia-insensitive mutants of the PUT4-encoded proline-specific permease in yeast. J. Gen. Appl. Microbiol. 55, 427-439, https://doi.org/10.2323/jgam.55.427 (2009).

45. Takagi, H., Taguchi, J. \& Kaino, T. Proline accumulation protects Saccharomyces cerevisiae cells in stationary phase from ethanol stress by reducing reactive oxygen species levels. Yeast 33, 355-363, https://doi.org/10.1002/yea.3154 (2016).

46. Shenton, D. et al. Global Translational responses to oxidative stress impact upon multiple levels of protein synthesis. J. Biol. Chem. 281, 29011-29021, https://doi.org/10.1074/jbc.M601545200 (2006)

47. Marullo, P., Bely, M., Masneuf-Pomarede, I., Aigle, M. \& Dubourdieu, D. Inheritable nature of enological quantitative traits is demonstrated by meiotic segregation of industrial wine yeast strains. FEMS Yeast Res. 4, 711-719, https://doi.org/10.1016/j. femsyr.2004.01.006 (2004).

48. Gao, D. et al. A survey of statistical software for analysing RNA-seq data. Hum. Genomics 5, 56, https://doi.org/10.1186/1479-73645-1-56 (2010).

49. Livak, K. J. \& Schmittgen, T. D. Analysis of relative gene expression data using real-time quantitative PCR and the $2^{-\Delta \Delta C T}$ method. Methods 25, 402-408 (2001).

50. León-Ruiz, V., Vera, S., González-Porto, A. V. \& San Andrés, M. P. Analysis of water-soluble vitamins in honey by isocratic RPHPLC. Food Anal. Method. 6, 488-496 (2013).

51. Redón, M., Guillamón, J. M., Mas, A. \& Rozès, N. Effect of lipid supplementation upon Saccharomyces cerevisiae lipid composition and fermentation performance at low temperature. Eur. Food Res. Technol. 228, 833-840 (2009).

52. Zhao, H., Li, J., Han, B., Li, X. \& Chen, J. Improvement of oxidative stress tolerance in Saccharomyces cerevisiae through global transcription machinery engineering. J. Ind. Microbiol. Biotechnol. 41, 869-878, https://doi.org/10.1007/s10295-014-1421-8 (2014).

\section{Acknowledgements}

This work was supported by the National Natural Science Foundation of China (Grant number 31601439; 31501458). We thank GENEWIZ Suzhou for the part of high throughput sequencing and bioinformatics analysis in this work.

\section{Author contributions}

H.W.Z. and J.Y.L. designed the study and K.L.Z. conducted the whole expriments and data analysis. J.Y.L. interpreted the RNA-seq data, and wrote the draft manuscript. H.W.Z. participated in the research coordination and revised this manuscript.

\section{Competing interests}

The authors declare no competing interests.

\section{Additional information}

Supplementary information is available for this paper at https://doi.org/10.1038/s41598-020-63631-2.

Correspondence and requests for materials should be addressed to H.Z.

Reprints and permissions information is available at www.nature.com/reprints.

Publisher's note Springer Nature remains neutral with regard to jurisdictional claims in published maps and institutional affiliations.

(c) (i) Open Access This article is licensed under a Creative Commons Attribution 4.0 International

License, which permits use, sharing, adaptation, distribution and reproduction in any medium or format, as long as you give appropriate credit to the original author(s) and the source, provide a link to the Creative Commons license, and indicate if changes were made. The images or other third party material in this article are included in the article's Creative Commons license, unless indicated otherwise in a credit line to the material. If material is not included in the article's Creative Commons license and your intended use is not permitted by statutory regulation or exceeds the permitted use, you will need to obtain permission directly from the copyright holder. To view a copy of this license, visit http://creativecommons.org/licenses/by/4.0/.

(C) The Author(s) 2020 